\title{
Finding optimal paths in MREP routing
}

\author{
Rudolf Fleischer ${ }^{\mathrm{a}, *}$, Mordecai Golin ${ }^{\mathrm{a}}$, Chin-Tau Lea ${ }^{\mathrm{b}, 1}$, Steven Wong ${ }^{\mathrm{b}}$ \\ a Department of Computer Science, HKUST, Clear Water Bay, Kowloon, Hong Kong \\ ${ }^{\mathrm{b}}$ Department of EEE, HKUST, Clear Water Bay, Kowloon, Hong Kong
}

Received 20 January 2003; received in revised form 14 October 2003

Communicated by K. Iwama

\begin{abstract}
Maximum Residual Energy Path (MREP) routing has been shown an effective routing scheme for energy conservation in battery powered wireless networks. Past studies on MREP routing are based on the assumption that the transmitting node consumes power, but the receiving node does not. This assumption is false if acknowledgment is required as occurs, for example, in some Bluetooth applications.

If the receiving node does not consume power then the MREP routing problem for a single message is easily solvable in polynomial time using a simple Dijkstra-like algorithm. We further show in that when the receiving node does consume power the problem becomes NP-complete and is even impossible to approximate with an exponential approximation factor in polynomial time unless $\mathrm{P}=\mathrm{NP}$.
\end{abstract}

() 2003 Elsevier B.V. All rights reserved.

Keywords: Approximation algorithms; Graph algorithms; Computational complexity

\section{Introduction}

Recent advances in wireless technologies, such as Bluetooth [10], have made it easy and practical to construct an ad hoc network for novel applicationsa surveillance network, a wireless tag network in a grocery store, and a sensor network to monitor environment dangerous conditions are just a few examples.

\footnotetext{
* Corresponding author.

E-mail addresses: rudolf@cs.ust.hk (R. Fleischer), golin@cs.ust.hk (M. Golin), eelea@ee.ust.hk (C.-T. Lea).

${ }^{1}$ Research is supported by the Hong Kong RGC grant HKUST6201/03E.
}

Battery power is a precious resource for each wireless device (except the wired gateway computer) and energy conservation is critical for such a network. Routing will play an important role in energy conservation. This issue has been studied extensively in the past $[1-3,9,11,14-16]$. A central part of any routing study is the definition of the path metric. Some metrics combine both delay and power consumption, whereas others focus on maximizing the system life time and ignore the delay.

When delay is less a concern than system life, Maximum Residual Energy Path (MREP) routing has been shown an effective scheme for energy conservation [24]. In MREP routing, the best path is one that maximizes the energy of that node on the path with the least energy after sending the message. Previous work 
on MREP routing concentrated on heuristics for a constant stream of messages to be routed through the network [2-4], the case of hybrid cost functions that try to balance total energy consumption and energy drain at a single node $[12,15]$, or just studied local routing heuristics in a distributed system [17]. All these studies have been based on the assumption that sending packets requires energy, but receiving packets does not. In this case, we show that the MREP routing problem for a single message is reducible to the maxbandwidth path problem and therefore polynomialtime solvable using a standard Dijkstra-type algorithm (a similar algorithm is sketched in [2]).

However, the assumption of energy-free reception is not always justified. Ad hoc networking technologies normally include energy-conservation states. Between two transmissions, nodes in the network may enter the energy-conservation state (sleeping) state. A sleeping node along the chosen path has to be waken up first. The process requires a hand-shaking packet exchanged between the sender and the receiver. Sending a hand-shaking packet by the receiver will cost energy. Another example is if the ad hoc network has very noisy connections (like an earthquake monitoring sensor network or a battlefield network), hop-byhop acknowledgment may be used. For every packet received, the receiver must send back an acknowledgment packet. This costs energy. We show that in this case the MREP routing problem for a single message is NP-complete and is impossible even to approximate with an exponential approximation factor in polynomial time unless $\mathrm{P}=\mathrm{NP}$.

After defining the MREP routing problem in Section 2, we will show in Section 3 that it can be efficiently solved in networks without acknowledgment costs by a simple transformation to the maxbandwidth path problem. We then show in Section 4 that the general problem with acknowledgment costs is NP-complete and very hard to approximate.

\section{Definitions}

We can model the MRE routing problem as follows. Let $G=(V, E)$ be a directed graph where $V$ is a set of vertices (wireless nodes) and $E$ is a set of directed edges (connections). Initially, each vertex $u$ has a battery charged with energy $E_{u} \geqslant 0$. This energy will be consumed by sending or acknowledging messages. We usually just speak of the energy of a node.

With each edge $e=(u, v)$ we associate two costs, the sending cost $s_{e}$ (or $s_{u, v}$ ) for sending a message along $e$, i.e., the power used up by the sender when transmitting the full message, and the acknowledgment $\operatorname{cost} r_{e}$ (or $r_{u, v}$ ) for receiving a message, i.e., the power used by the receiver to transmit an acknowledgment message back to the sender. Sending a message along $e=(u, v)$ will reduce $E_{u}$, the energy of $u$, by $s_{e}$, and it will reduce $E_{v}$ by $r_{e}$. Of course, sending a message is only possible if neither node energies become negative. Normally, $s_{e}$ and $r_{e}$ will depend on the distance between the vertices and on the size of the message (in our definition we assume all messages have unit size), but that is not important for our results.

When sending a message from a vertex $s$ to a vertex $t$ in $G$ we can usually choose between many different paths along which we could route the message. We are interested in paths that leave a high energy in all the nodes on the path, i.e., we try to avoid situations where routing a message would use up all the energy of one node. Otherwise, we might have trouble routing the next message (the network could even become disconnected).

Formally, if we route a message along a path $P=\left(v_{1}, v_{2}, \ldots, v_{k-1}, v_{k}\right)$ in $G$, where $v_{1}, \ldots, v_{k}$ are vertices and $\left(v_{1}, v_{2}\right), \ldots,\left(v_{k-1}, v_{k}\right)$ are edges, then each node on $P$ loses some energy due to the send and acknowledgment costs. Let $R_{u}$ denote the residual energy of $u$ after routing the message. If $P$ is simple then $R_{v_{1}}=E_{v_{1}}-s_{v_{1}, v_{2}}, R_{v_{i}}=E_{v_{i}}-$ $r_{v_{i-1}, v_{i}}-s_{v_{i}, v_{i+1}}$ for $i=2, \ldots, k-1$, and $R_{v_{k}}=$ $E_{v_{k}}-r_{v_{k-1}, v_{k}}$. Otherwise, $P$ contains some vertex (or vertices) several times, and then we have to add up all the send and acknowledgment costs for that vertex. We now define the minimum residual energy (MRE) of $P$, denoted by $D(P)$, to be

$$
D(P)=\min _{i=1, \ldots, k}\left\{R_{v_{i}}\right\} .
$$

Note that we can only send a message along $P$ if all nodes on $P$ have non-negative residual energy, i.e., $D(P) \geqslant 0$. We call such a path legal. See Fig. 1 for an example.

We call a path optimal if it has maximum MRE among all paths that route a message from a given start vertex to a given end vertex. Routing along a nonsimple path is obviously never a good idea, so 


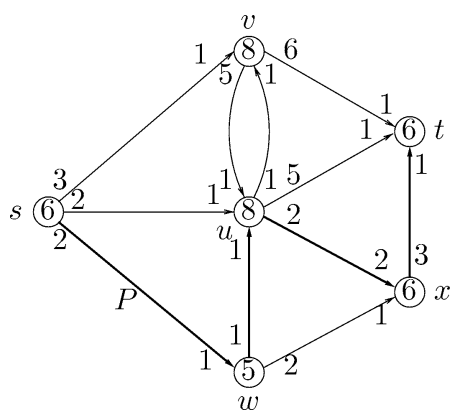

Fig. 1. A routing network. Nodes are labeled by their energy. Outgoing edges are labeled by the send cost along the edge, and incoming edges are labeled by the acknowledgment cost. The path $P=(s, w, u, x, t)$ has $D(P)=1$ (with node $x$ being the bottleneck) and is therefore legal.

optimal paths are always simple. Our goal is to find optimal $s-t$ paths.

\section{MREPP without acknowledgment costs}

\subsection{The max-bandwidth problem}

In this section we quickly review the max-bandwidth problem for graphs with bandwidth constraints on the edges and its solution. We will present an algorithm that will be used in the next section as a subroutine to solve MREPP without acknowledgment costs.

Let $G=(V, E)$ be a directed graph such that associated with each edge $(u, v) \in E$ there is a bandwidth $b(u, v) \geqslant 0$. Let $P=\left(v_{1}, v_{2}, \ldots, v_{k-1}, v_{k}\right)$ be a path in $G$. The bandwidth of $P$ is $B(P)=\min _{1 \leqslant i<k} b\left(v_{i}\right.$, $v_{i+1}$ ), i.e., the smallest bandwidth among all edges on $P$ (also called the bottleneck of $P$ ). Given $s, t \in V$, the max-bandwidth problem is to find a path from $s$ to $t$ that maximizes $B(P)$. This problem has been well studied and it is well known that such a path can be found using a simple modification of Dijkstra's shortest path algorithm [5,6]. A good history and de- scription of the modified algorithm using modern notation is available in [13]. The standard implementation of this algorithm runs in $\mathrm{O}(|E| \log |V|)$ time; with more sophisticated data structures this can be further reduced to $\mathrm{O}(|E|+|V| \log |V|)$ [7].

\subsection{Solving MREPP}

In this section we describe how to find optimal MRE paths when there are no acknowledgment costs for messages, i.e., $r_{e}=0$ for all edges $e$. To solve this problem we run the max-bandwidth algorithm from the previous section on the graph obtained from the given network by defining the bandwidth of an edge as the residual energy that would remain in the node if we used the edge to route the message.

Assume we want to find an optimal $s-t$ path, where $s$ and $t$ are nodes of the given network $G=$ $(V, E)$. Without acknowledgment costs, the residual energy of a node $v_{i}$ on an $s-t$ path $P=\left(s=v_{1}\right.$, $\left.v_{2}, \ldots, v_{k-1}, v_{k}=t\right)$ is $R_{v_{i}}=E_{v_{i}}-s_{v_{i}, v_{i+1}}$, for $i=1$, $\ldots, k-1$, and it is $R_{t}=E_{t}$ for the node $t$. We now transform $G$ into a new graph $G^{\prime}$ by adding a new node $t^{\prime}$ and a new edge $\left(t, t^{\prime}\right)$ with bandwidth equal to $E_{t}$. For all other edges $(u, v)$ of $G^{\prime}$ we define the bandwidth to be $b(u, v)=E_{u}-s_{u, v}$. Fig. 2(a) shows the transformed graph of Fig. 1 (when all acknowledgment costs are set to zero).

Obviously, there is a one-to-one correspondence between $s-t$ paths in $G$ and $s-t^{\prime}$ paths in $G^{\prime}$. Moreover, if $P$ is an $s-t$ path in $G$ and $P^{\prime}$ is the corresponding $s-t^{\prime}$ path in $G^{\prime}$ then $D(P)=B\left(P^{\prime}\right)$. For example, in Fig. 1 the path $P=(s, u, t)$ has $D(P)=3$ (ignoring the acknowledgment costs), and the corresponding path $P^{\prime}=\left(s, u, t, t^{\prime}\right)$ has $B\left(P^{\prime}\right)=3$.

To find the max-bandwidth path in $G^{\prime}$, we can use the max-bandwidth algorithm from the previous section. The tree path from $s$ to $t^{\prime}$ corresponds to the optimal MRE $s-t$ simple path in $G$. In Fig. 3 we summarize the algorithm to compute an optimal MRE path for networks without acknowledgment costs.

MREPP (Maximum Residual Energy Path Problem)

Input: A directed graph $G=(V, E)$ with vertex energies $E_{u}$, and send costs $s_{u, v}$ and acknowledgment costs $r_{u, v}$ on the edges $(u, v)$. There are also two distinguished vertices $s$ (source) and $t$ (destination).

Output: An $s-t$ path in $G$ that maximizes $D(P)$ among all $s-t$ paths $P$ in $G$. 


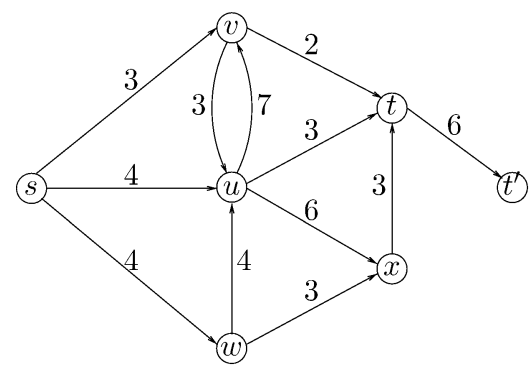

(a)

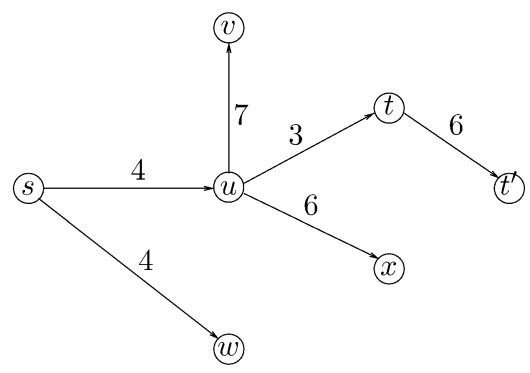

(b)

Fig. 2. (a) The network of Fig. 1 with zero acknowledgment costs and residual node energies replaced by bandwidth constraints on the edges. There are several max-bandwidth $s-t^{\prime}$ paths of cost 3 . One such path is $s, u, t, t^{\prime}$, with bottleneck $(u, t)$. (b) The max-bandwidth spanning tree computed by the modified Dijkstra's algorithm. Note that every path in the tree from $s$ to a node $w$ is a max-bandwidth $s-w$ path.

1. Transform $G=(V, E)$ into $G^{\prime}=\left(V^{\prime}, E^{\prime}\right)$ by adding vertex $t^{\prime}$ and edge $\left(t, t^{\prime}\right)$. Set $b(u, v)=E_{u}-s_{u, v}$ for edges $(u, v) \in E$, and $b\left(t, t^{\prime}\right)=E_{t}$.

2. Use a max-bandwidth algorithm to find $P^{\prime}$, a max-bandwidth $s-t^{\prime}$ path in $G^{\prime}$.

3. Return $P$, the $s-t$ path in $G$ corresponding to $P^{\prime}$.

Fig. 3. Algorithm for solving MREPP in networks without acknowledgment costs.

For example, running the modified Dijkstra algorithm on the transformed graph in Fig. 2(a), gives the spanning tree in Fig. 2(b). In this tree, the maxbandwidth $s-t^{\prime}$ path is $P^{\prime}=\left(s, u, t, t^{\prime}\right)$ with bandwidth $B\left(P^{\prime}\right)=3$. Thus, an optimal MRE $s-t$ path in the graph in Fig. 1 is $P=(s, u, t)$ with $D(P)=3$ (ignoring acknowledgment costs).

\section{The NP-completeness proof}

In this section we show that MREPP is NP-complete. We actually show that even deciding whether there exists a legal routing path between two specified nodes is NP-complete. Later we will slightly modify the construction in the proof of the theorem to show that approximating MREPP is also very difficult.

We will use reduction from 3-SAT, so we first quickly review the 3-SAT problem [8]. An instance of 3-SAT is a set $U=\left\{x_{1}, x_{2}, \ldots, x_{n}\right\}$ of variables and a formula which is given as a collection $C_{1}, C_{2}, \ldots, C_{m} \subseteq 2^{U}$ of clauses. A literal is either a variable $x_{i}$ or its negation $\bar{x}_{i}$; in the former case the literal is positive, in the latter case it is negative. Each clause $C_{j}=\left\{\ell_{j, 1}, \ell_{j, 2}, \ell_{j, 3}\right\}$ consists of three literals. A truth assignment is a function $\phi: U \rightarrow\{T, F\}$. A positive literal $x_{i}$ is satisfied by $\phi$ if $\phi\left(x_{i}\right)=T$, a negative literal $\bar{x}_{i}$ is satisfied if $\phi\left(x_{i}\right)=F$. A clause is satisfied if at least one of its literals is satisfied. A formula is satisfiable if there is a truth assignment that satisfies all of its clauses. Deciding whether such an assignment exists is NP-complete.

\section{Theorem 1. MREPP is NP-complete.}

Proof. Since we can easily check whether a given routing leaves at least a specified minimum residual energy in the nodes along the routing path, MREPP is in NP.

We will prove that MREPP is NP-hard by showing a polynomial-time reduction from 3-SAT to MREPP. That is, given an instance of 3-SAT we will in polynomial time construct an instance of MREPP that has a legal path from $s$ to $t$ if and only if the 3-SAT instance is satisfiable.

We now describe how to transform an instance of 3-SAT to an instance of MREPP. Let the formula $F$ be given as a set of $m$ clauses $C_{j}=\left\{\ell_{j, 1}, \ell_{j, 2}, \ell_{j, 3}\right\}$, for $j=1, \ldots, m$, over $n$ variables $x_{1}, \ldots, x_{n}$. We construct a directed graph as in Fig. 4. It contains two kinds of gadgets. For each variable $x_{i}, i=1, \ldots, n$, we have the variable gadget $V_{i}$ (see Fig. 5). In this gadget, the message can be routed along one of two parallel paths. If the message is routed along the upper 


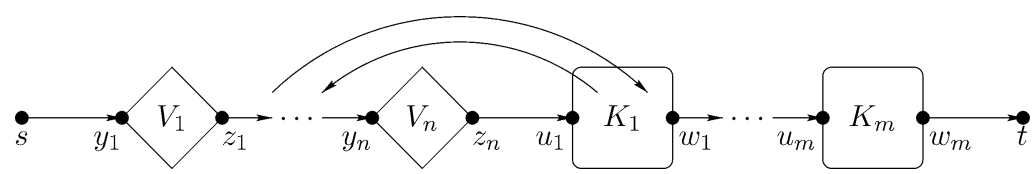

Fig. 4. The NP-completeness reduction from 3-SAT. We try to send a message from $s$ to $t$. This is possible if and only if the given 3-SAT formula is satisfiable. The connections between the gadgets $V_{i}$ and $K_{j}$ are only indicated.

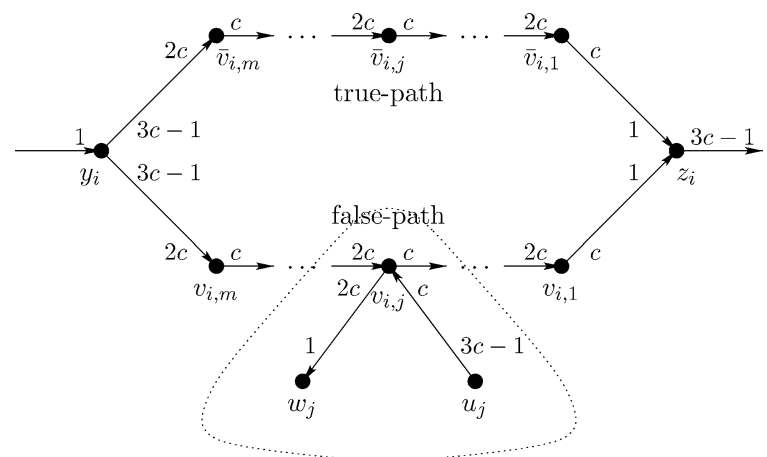

Fig. 5. The variable gadget $V_{i}$. Routing the message along the upper (lower) path corresponds to setting $x_{i}$ to $T(F)$. The dotted line shows the interaction with clause $C_{j}$ if that clause contains the literal $x_{i}$.

path in the figure then we interpret this as setting $x_{i}$ to $T$; therefore, we call this path the true-path. Similarly, the lower path corresponds to the setting $x_{i}=F$, so this path is called the false-path.

After the message has passed all the variable gadgets (and all variables have been assigned a truth value) it must be routed through the clause gadgets $K_{j}$, for $j=1, \ldots, m$. To satisfy a clause, we must route the message through one of three parallel paths, each corresponding to one of the literals of the clause (see Fig. 6). Each of these paths moves back to a node in the variable gadget of the respective literal. If the clause contains the positive literal $x_{i}$ then the clause gadget connects to the node $v_{i, j}$ on the false-path and if the clause contains the negative literal $\bar{x}_{i}$ then the clause gadget connects to the node $\bar{v}_{i, j}$ on the truepath. Choosing the node energies and the send and acknowledgment costs appropriately (we will do this later), each node in a variable gadget can route the message at most once. Of course, if the formula is satisfiable then we can always route the message along the path corresponding to the literal in the clause that is true in the satisfying assignment. Thus, the existence of a satisfying assignment for the formula induces an $s-t$ routing path through the network. If all the

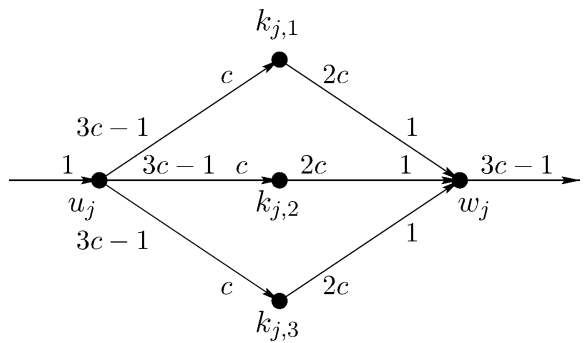

Fig. 6. The clause gadget $K_{j}$. If $\ell_{j, p}$, for $p=1,2,3$, is the variable $x_{i}$ then $k_{j, p}$ is the node $v_{i, j}$ in $V_{i}$; otherwise, it is the node $\bar{v}_{i, j}$.

nodes have sufficient energy for routing the message then this path is also legal. Fig. 7 shows the complete network for the formula $\left(x_{1} \vee \bar{x}_{2} \vee x_{3}\right) \wedge\left(\bar{x}_{1} \vee \bar{x}_{2} \vee\right.$ $\left.x_{3}\right)$, together with the $s-t$ path corresponding to the satisfying truth assignment $x_{1}=T, x_{2}=F$, and $x_{3}=T$.

It remains to show that every $s-t$ path induces a satisfying assignment for the formula. This can be guaranteed by a clever choice of the node energies and the send and acknowledgment costs for the edges. Let $c \geqslant 1$ be fixed. For the correctness of our reduction, the actual value of $c$ is not important. But to get a polynomial-time reduction we must restrict $c$ to a value that can be computed in polynomial time.

All nodes have energy $4 c-1$. The edge $\left(s, y_{1}\right)$ has send cost $s_{s, y_{1}}=3 c$ and acknowledgment $\operatorname{cost} r_{s, y_{1}}=$ 1. The edge $\left(w_{m}, t\right)$ also has send cost $s_{w_{m}, t}=3 c$ and acknowledgment cost $r_{w_{m}, t}=1$.

In the variable gadgets $V_{i}$, for $i=1, \ldots, n$, the incoming edge at $y_{i}$ has acknowledgment cost 1 , and the outgoing edges of $y_{i}$ have send cost $s_{y_{i}}, \bar{v}_{i, m}=$ $s_{y_{i}, v_{i, m}}=3 c-1$ and acknowledgment cost $r_{y_{i}, \bar{v}_{i, m}}=$ $r_{y_{i}, v_{i, m}}=2 c$. The incoming edges of $z_{i}$ have send cost $s_{\bar{v}_{i, 1}, z_{i}}=s_{v_{i, 1}, z_{i}}=c$ and acknowledgment cost $r_{\bar{v}_{i, 1}, z_{i}}=r_{v_{i, 1}, z_{i}}=1$, and the outgoing edge at $z_{i}$ has send cost $3 c-1$. All other edges on the true-path and the false-path have send cost $s_{\bar{v}_{i, j}, \bar{v}_{i, j-1}}=s_{v_{i, j}, v_{i, j-1}}=$ $c$ and acknowledgment cost $r_{\bar{v}_{i, j}, \bar{v}_{i, j-1}}=r_{v_{i, j}, v_{i, j-1}}=$ $2 c$, for $j=m, \ldots, 2$. Thus, routing through $V_{i}$ leaves 


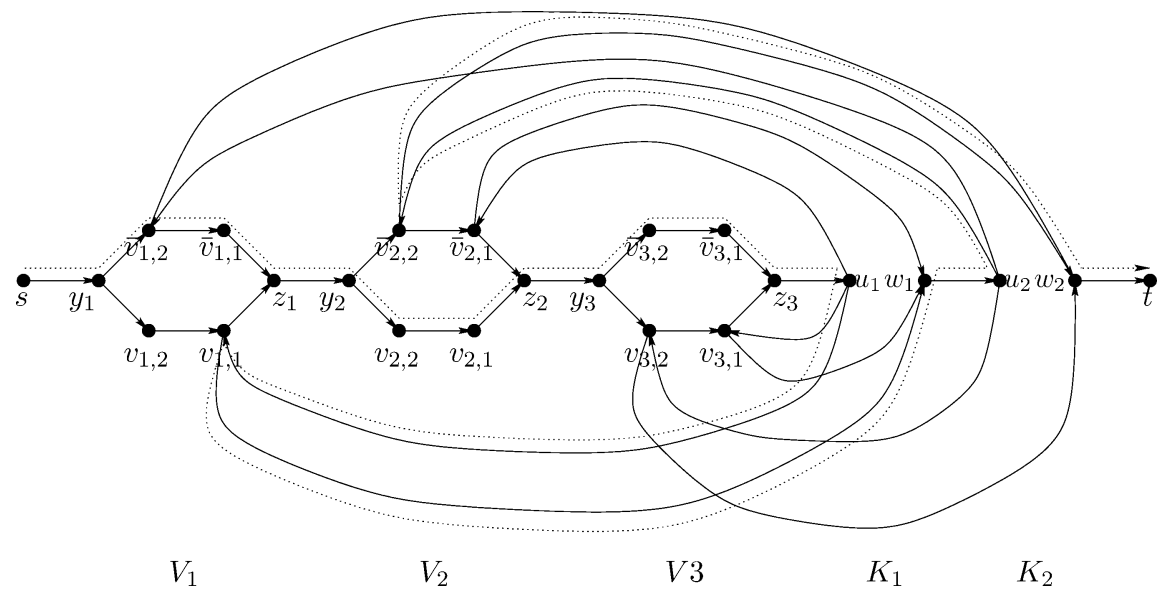

Fig. 7. The network we construct for the formula $\left(x_{1} \vee \bar{x}_{2} \vee x_{3}\right) \wedge\left(\bar{x}_{1} \vee \bar{x}_{2} \vee x_{3}\right)$. The dotted line is the legal $s-t$ path corresponding to the satisfying truth assignment $x_{1}=T, x_{2}=F$, and $x_{3}=T$.

residual energy $c-1$ in all nodes on the routing path (which is not enough to route the message a second time).

In the clause gadgets $K_{j}$, for $j=1, \ldots, m$, the incoming edges of $u_{j}$ have acknowledgment cost 1 , and the outgoing edges have send cost $s_{u_{j}, k_{j, 1}}=$ $s_{u_{j}, k_{j, 2}}=s_{u_{j}, k_{j, 3}}=3 c-1$. The incoming edges at $w_{j}$ have acknowledgment cost $r_{k_{j, 1}, w_{j}}=r_{k_{j, 2}, w_{j}}=$ $r_{k_{j, 3}, w_{j}}=1$, and the outgoing edges have send cost $3 c-1$. Thus, routing through $K_{j}$ leaves residual energy $c-1$ in all nodes on the routing path.

It is now easy to see that there is no legal $s-t$ path that does not correspond to a satisfying assignment. Consider an arbitrary legal $s-t$ path $P . P$ must enter the gadget $V_{i}$ at $y_{i}$, for $i=1, \ldots, n$, follow either the true-path or the false-path (because the nodes do not have enough energy to send the message along one of the edges going to a node in a clause gadget), and leave $V_{i}$ at node $z_{i}$. After the last gadget $V_{n}$, all nodes $z_{i}$ have residual energy $c-1$, so $P$ cannot pass through these nodes again later.

Now $P$ enters the first clause gadget $K_{1}$. It can only follow an edge to a node $v$ in some $V_{i}$ that was not used before because the nodes in $V_{i}$ do not have enough energy to receive and send two messages (or the same message twice). From $v$, which is either $v_{i, 1}$ or $\bar{v}_{i, 1}$, the message cannot be routed to $z_{i}$ again (because the message passed already once through $z_{i}$ ). So it must go back to $K_{1}$ from $v$, i.e., it goes to $w_{1}$. But then $P$ has satisfied clause $C_{1}$ because $v$ corresponds to a true literal. Note that this argument is the reason why we ordered the nodes on the true-path (and falsepath) in reverse order.

From $w_{1}, P$ enters the second clause gadget $K_{2}$. Again, it must satisfy the clause by choosing an edge to either $v_{i, 2}$ or $\bar{v}_{i, 2}$ in some $V_{i}$ that corresponds to a true literal. And again, it must immediately return to $K_{2}$ because otherwise it would have to leave $V_{i}$ either via $z_{i}$ or via $w_{1}$ (if $x_{1}$ is also a literal in $C_{1}$ ), and these both cases are not possible. Continuing this argument, we can prove by induction on $j=1, \ldots, m$ that $P$ must enter $K_{j}$ at $u_{j}$ and leave it at $w_{j}$, so $P$ indeed induces a satisfying assignment for all clauses. Also, all nodes on $P$ will have energy $c-1$ after the routing, so we have $D(P)=c-1$. Thus, $P$ is legal if $c$ is at least 1 .

Finally, we must show that our reduction is polynomial-time. Our network has $\mathrm{O}(\mathrm{nm})$ nodes and $\mathrm{O}(\mathrm{nm})$ edges that can all be computed in polynomial time from the given description of the formula. Also, all edge costs and node energies can be computed in polynomial time if $c=\mathrm{O}\left(2^{\text {poly }(n m)}\right)$. Choosing $c=1$ actually suffices for the correctness of the construction. We need other values of $c$ later in the proof of non-approximability.

In the proof of the theorem above, some of the edges have a higher acknowledgment than send cost. If this was not allowed in a more restrictive (but more realistic) routing scenario we could modify the construction by adding a new node with energy $4 c-1$ 
in the middle of each edge, where the incoming edge has acknowledgment cost 1 and the outgoing edge has send cost $3 c-1$.

With another slight modification of the construction in the proof above we can also prove that approximating MREPP is very difficult.

Theorem 2. MREPP cannot be approximated within a factor of $\mathrm{O}\left(2^{\text {poly }(n)}\right)$ in polynomial time unless $\mathrm{P}=$ $\mathrm{NP}$, where $n$ is the number of nodes in the network.

Proof. We prove the theorem by showing that a polynomial-time approximation algorithm for MREPP with an approximation ratio of $\rho=\mathrm{O}\left(2^{\text {poly }(n)}\right)$ would enable us to solve 3-SAT in polynomial time. We use essentially the same construction as in the proof of Theorem 1. We just add one more edge from $s$ to $t$, with send cost and acknowledgment cost $4 c-2$.

In this network, there always exists a legal $s-t$ path with minimum residual energy 1 , namely the edge $(s, t)$. Another path of minimum residual energy $c$ exists if and only if the formula $F$ represented by the network is satisfiable. Since we could choose $c=2 \rho=\mathrm{O}\left(2^{\text {poly }(n)}\right)$, any approximation algorithm for MREPP with approximation $\rho$ could decide the satisfiability of $F$.

\section{Conclusions}

We have shown that MREPP is a very difficult problem in networks with acknowledgment costs. But this is only the problem of routing a single message. What we actually want is a good routing scheme for many messages, either in an online setting (and then we would like to use either competitive analysis or some probabilistic analysis) or in a situation where we have a batch of messages for which we want to find a good routing schedule. Since these problems seem to be rather hard, we might concentrate on finding heuristics that work well in practice.

\section{References}

[1] D. Bertsekas, R. Gallager, Data Networks, 2nd edn., PrenticeHall, Englewood Cliffs, NJ, 1987.
[2] J.-H. Chang, L. Tassiulas, Routing for maximum system lifetime in wireless ad-hoc networks, in: Proc. 37th Annual Allerton Conf. on Communication, Control, and Computing, Monticello, IL, September 1999.

[3] J.-H. Chang, L. Tassiulas, Energy conserving routing in wireless ad-hoc networks, in: Proc. 19th Annual IEEE Conf. on Computer Communications (INFOCOM 2000), Tel Aviv, Israel, Vol. 1, March 2000, pp. 22-31.

[4] J.-H. Chang, L. Tassiulas, Fast approximate algorithms for maximum lifetime routing in wireless ad-hoc networks, in: Networking, in: Lecture Notes in Comput. Sci., Vol. 1815, Springer, Berlin, 2000, pp. 702-713.

[5] T. Cormen, C. Leiserson, R. Rivest, Introduction to Algorithms, McGraw-Hill/MIT Press, New York/Cambridge, MA, 1990.

[6] J. Edmonds, R.M. Karp, Theoretical improvements in algorithmic efficiency for network flow problems, J. ACM 19 (2) (1972) 248-264.

[7] M. Fredman, R. Tarjan, Fibonacci heaps and their uses in improved network optimization problems, J. ACM 34 (1987) 596-615.

[8] M.R. Garey, D.S. Johnson, Computers and Intractability: A Guide to the Theory of NP-Completeness, W.H. Freeman and Company, New York, 1979.

[9] J. Gomez, A.T. Campbell, M. Naghshineh, C. Bisdikian, Power aware routing in wireless packet networks, in: Proc. 6th Internat. Workshop on Mobile Multimedia Communications (MoMuC'99), San Diego, CA, November 1999, pp. 380-383.

[10] J.C. Haartsen, S. Mattisson, Bluetooth-A new low-power radio interface providing short-range connectivity, IEEE Proc. 88 (10) (2000) 1651-1661.

[11] D. Johnson, D. Maltz, Dynamic source routing in ad hoc wireless networks, in: T. Imielinski, H. Korth (Eds.), Mobile Computing, Chapter 5, Kluwer Academic Publishers, Dordrecht, 1996, pp. 153-181.

[12] K. Kalyan Kumar, A. Chockalingam, Energy efficient routing in wireless ad-hoc networks, URL http://citeseer.nj.nec.com/ 523912.html.

[13] N. Malpani, J. Chen, A note on practical constructions of maximum bandwidth paths, 2002, URL http://www.cs.tamu.edu/ course-info/cpsc629/chen/notes/.

[14] S. Murthy, J.J. Garcia-Luna-Aceves, An efficient routing protocol for wireless networks, Mobile Networks Appl. 1 (2) (1996) 183-197.

[15] S. Singh, M. Woo, C.S. Raghavendra, Power-aware routing in mobile ad hoc networks, in: Proc. 4th Annual Internat. Conf. on Mobile Computing and Networking (MOBICOM'98), Dallas, TX, October 1998, pp. 181-190.

[16] I. Stojmenovic, X. Lin, Power aware localized routing in wireless networks, in: Proc. 14th IEEE Internat. Parallel and Distributed Processing Symposium (IPDPS'00), Cancun, Mexico, May 2000, pp. 371-376.

[17] K. Woo, C. Yu, D. Lee, H.Y. Youn, B. Lee, Non-blocking, localized routing algorithm for balanced energy consumption in mobile ad hoc networks, URL http://citeseer.nj.nec.com/ 510965.html. 